\title{
Non-trophic functions of carcasses: from death to the nest
}

$\mathrm{F}$ rom 2010 to 2012, we had the unforgettable experience of living and working in the Zululand region of South Africa, studying the diverse community of vertebrate scavengers inhabiting the savanna. Our fieldwork was mainly conducted in two neighboring protected areas: Hluhluwe-iMfolozi Park and Mkhuze Game Reserve. We monitored the consumption of nearly 100 carcasses - everything from domestic chickens to ungulates ranging from impalas (Aepyceros melampus) to elephants (Loxodonta africana) - using motion-sensing camera traps (see Moleón et al. 2015 for details). To obtain the relevant data on scavenging, we separated the photographs of scavengers from those in which no scavenger species appeared. But upon further inspection, the images without scavengers revealed an interesting behavior. One photograph in particular attracted our attention. It showed a black Cape glossy starling (Lamprotornis nitens) with a white feather in its beak; the starling was perched on a bush above a chicken carcass (Figure 1). This brought to mind a paper we had published with our colleague Lola García-López de Hierro a decade previously, about the ecological and evolutionary importance of the carrying of feathers to nests by male house sparrows (Passer domesticus), a study also set in South Africa (García-López de Hierro et al. 2013). Many bird species use feathers for nest

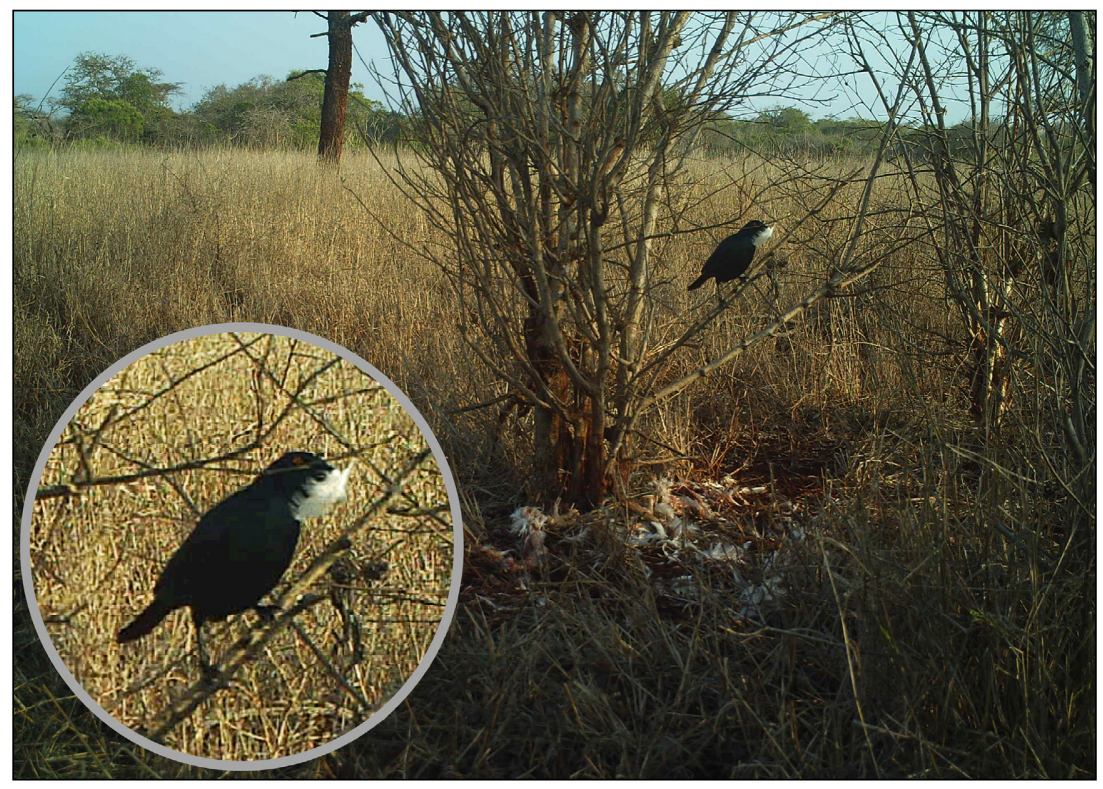

Figure 1. A Cape glossy starling (Lamprotornis nitens) collects a feather from a chicken carcass that had been scavenged by a large-spotted genet (Genetta tigrina) and white-tailed mongoose (Ichneumia albicauda); note the scattering of feathers resulting from the scavenging process. Photograph was taken with a motion-sensing camera in Mkhuze Game Reserve, South Africa. construction and decoration, and feather-carrying behavior is often involved in sexual selection because it can be an indication of individual male quality (Mainwaring et al. 2014). Our photograph led us to wonder whether bird carcasses or feathers left at carcass sites by avian scavengers might be a common source of feathers for other birds.

Cape glossy starlings add a lining of feathers to their nests during egg incubation (Hockey et al. 2005), but little is known about where the starlings obtain these feathers. To shed some light on this question, we examined our other photos. For each carcass observed during the starling breeding season (August-May), we noted the presence or absence of starlings in each photo. Where starlings were present, we also recorded the number of individuals present and whether they collected any feathers. We divided images of ungulate carcasses (14 in Hluhluwe-iMfolozi and 17 in Mkhuze) into two categories: carcasses visited - or not visited by vultures. While feeding on ungulate carcasses, vultures typically gather in large numbers; during this time, they often shed down and feathers, which could be a source of nest-lining material for starlings in the vicinity. In the case of photos associated with chicken carcasses (21 in Hluhluwe-iMfolozi and 18 in Mkhuze), we noted whether those carcasses had been visited - or not visited - by unspecified scavengers before the arrival of the starlings. For a relatively small bird such as a starling, chicken feathers are much easier to obtain after the body of the carcass has been opened and the feathers separated from the skin (Figure 1).

We found that starlings regularly visited both carcass types and took feathers. In addition, most visits and all feathercollecting events happened after scavengers had visited the carcass (WebFigure 1). Our findings seemed to indicate that carcasses are indeed a source of feathers for starlings, and that the actions of the scavengers (vultures in the case of ungulate carcasses, and any scavenger in the case of chicken carcasses) are vital in facilitating feather availability, at least for these small birds. This could be considered a new type of commensalism, with wide ecological, evolutionary, and conservation implications (vultures and other major scavengers are under threat worldwide), which therefore deserve indepth scientific attention. 

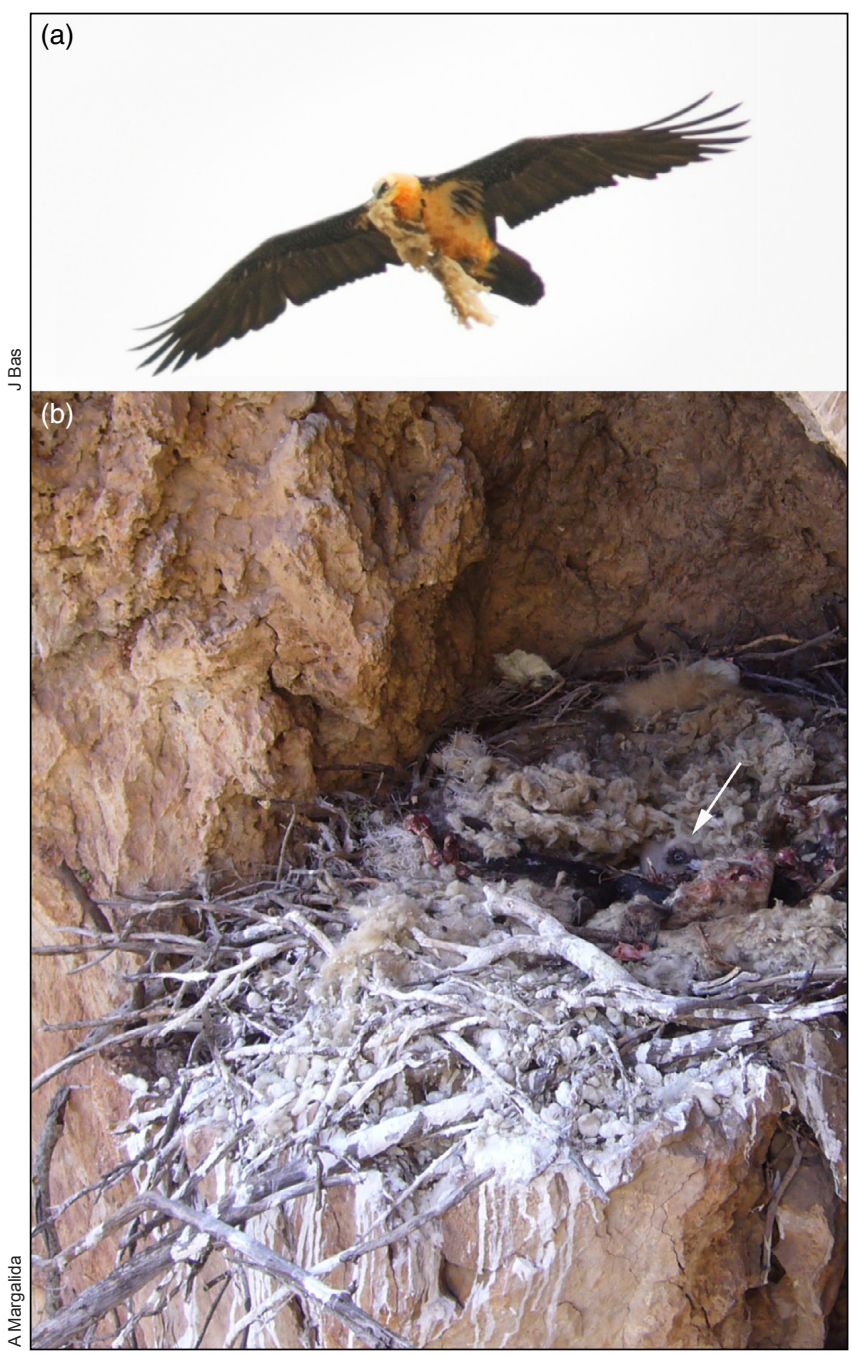

Figure 2. (a) An adult bearded vulture (Gypaetus barbatus) carrying wool from a sheep carcass to the nest. (b) An active bearded vulture nest containing a small chick (arrow), and showing the abundant wool lining. Photographs both taken in the Spanish Pyrenees.

Carcasses are already known to provide nest construction materials for other avian species, including bearded (Gypaetus barbatus; Figure 2) and Egyptian (Neophron percnopterus) vultures (Donázar 1993). Such materials range from wool to feathers, skin, bones, and hooves. These vultures benefit from the carcasses both as a food source and as a source of nest materials. Unlike the starling, the action of other scavengers is not required in these cases because vultures are powerful enough to tear off the nesting material themselves. Overall, these observations indicate that carcasses may play a more diverse and important role than generally recognized. Traditionally, ecologists have approached carcass research mostly from a trophic point of view, focusing on their role in nutrient cycling through direct and indirect interactions (Danell et al. 2002; DeVault et al. 2003; Wilson and Wolkovich 2011; Moleón et al. 2014a). However, carcasses have several potential non-trophic ecological and evolutionary functions apart from providing food for scavengers or serving as a hotspot of nutrients for microorganisms and plants. For instance, carcasses are a refuge and habitat for certain invertebrates (Selva and Cortés-Avizanda 2009), are important for the dynamics of non-trophically transmitted diseases (Samuel et al. 2001; Turner et al. 2014), and have for millennia provided raw material to humans for tools and other instruments (Moleón et al. 2014b). Many opportunities exist for further inquiries into the multiple non-trophic functions of carcasses, which may be fruitful not only for ecologists but also for evolutionary biologists, anthropologists, paleontologists, conservation biologists, and wildlife management practitioners. Our experience in South Africa demonstrates how chance findings based on systematic monitoring schemes can illuminate new and promising research avenues.

\section{Acknowledgements}

We thank N Owen-Smith for his continued support. This study was partly funded by the Spanish Ministry of Economy and Competitiveness through project CGL2012-40013-C02-02. MM was supported by a postdoctoral grant from the Spanish Ministry of Education (Plan Nacional de I+D+i 2008-2011) during his stay in South Africa, and currently by the Severo Ochoa Program for Centres of Excellence in $\mathrm{R}+\mathrm{D}+\mathrm{I}$ (SEV-2012-0262). JL Tella provided helpful comments on an earlier version of the manuscript. $\mathrm{J}$ Bas and A Margalida provided the photographs for Figure 2.

\section{Supporting Information}

Additional, web-only material, including the References for this article, may be found in the online version of this article at http://onlinelibrary.wiley.com/doi/10.1002/ fee.1306/suppinfo

\section{Marcos Moleón ${ }^{1,2,3 *}$ and José A Sánchez-Zapata ${ }^{2}$}

${ }^{1}$ Centre for African Ecology, School of Animal, Plant and

Environmental Sciences, University of the Witwatersrand, Johannesburg, South Africa;

${ }^{2}$ Department of Applied Biology, University Miguel Hernández, Alicante, Spain *(mmoleonpaiz@hotmail.com); ${ }^{3}$ Present address: Department of Conservation Biology, Doñana Biological Station (EBD-CSIC), Seville, Spain 